\title{
PERPENDICULAR MAGNETIC ANISOTROPY AND MAGNETO-OPTICAL KEER PROPERTIES OF Co/(Pd-Au) METALLIC MULTILAYERS
}

\author{
M. Kamiko, J.-G. Ha, K. Kyuno and R. Yamamoto \\ Institute of Industrial Science, University of Tokyo \\ 7-22-1, Roppongi, Minato-ku, Tokyo 106, JAPAN
}

\begin{abstract}
We prepared $\mathrm{Co} /(\mathrm{Pd}-\mathrm{Au})$ multilayers onto a glass substrates by if magnetron sputtering at room temperature. $\mathrm{Co} /(\mathrm{Pd}-$ $\mathrm{Au}$ ) multilayers exhibit a magnetic easy axis perpendicular to the film plane up to a larger magnetic layer thickness than $\mathrm{Co} / \mathrm{Pd}$ multilayer. Especially, $\mathrm{Co} /\left(\mathrm{Pd}_{0.70} \mathrm{Au}_{0.30}\right)$ multilayers became perpendicular at $33 \AA$. The magnetic anisotropy can be explained by the strain effect. The role of magnetoelastic energy due to the in-plane strain in the system is pointed out and discussed. We also investigated the magneto-optical(MO) polar Kerr effect of these multilayers. The polar Kerr rotation angle qk increases when the Au concentration is 4.5 at $\%$ compared to Co/Pd multilayers, but decreases with increasing Au content. KEYWORDS: PERPENDICULAR MAGNETIC ANISOTROPY, MAGNETO-OPTICAL KEER EFFECT, Co/(Pd-Au) MULTILAYERS.
\end{abstract}

\section{INTRODUCTION}

The magnetic and magneto-optical properties of metallic multilayers are of great interest both from fundamental and technological viewpoints. Metallic multilayers offer several potential advantages. Stable metal constituents with high corrosion and oxidation resistance can be selected, avoiding the necessity for protective layers. Enhancements in the polar Kerr rotation have been reported near the plasma frequency of the nonmagnetic metal in several multilayers such as $\mathrm{Co} / \mathrm{Au}$, $\mathrm{Co} / \mathrm{Cu}$ multilayers[1], and near the shorter-wavelength range(about $400 \mathrm{~nm}$ ) in $\mathrm{Co} / \mathrm{Pt}, \mathrm{Co} / \mathrm{Pd}$ multilayers[2].

As has been reported in several systems, $\mathrm{Co} / \mathrm{Pd}, \mathrm{Co} / \mathrm{Au}$, and $\mathrm{Co} / \mathrm{Pt}$ the magnetic layer is limited a few monolayers [3][4][5][6]. Namely, $\mathrm{Co} / \mathrm{Pd}$ and $\mathrm{Co} / \mathrm{Pt}$ multilayers have an easy axis of magnetization perpendicular to the thin-film plane, only when the Co layers are very thin $\left(t_{c o}<8 \AA\right.$ in $\mathrm{Co} / \mathrm{Pd}$ and $t_{c o}<$ $14 \AA$ in $\mathrm{Co} / \mathrm{Pt})[4][5]$. The origin of magnetic perpendicular anisotropy has been believed to be related with the change in the magnetic anisotropy of the interfacial atoms as a consequence of a reduced symmetry in their surroundings first suggested by Néel[7]. However, recent researches have indicated that perpendicular anisotropy can not be explained only by the Néel's surface anisotropy. Moreover, strain at magnetic-nonmagnetic interfaces have been suggested as possible origins, especially for the samples made by sputtering[8][9][10].
Stress and strain induced by the lattice mismatch between magnetic and non-magnetic layers can be used to change the properties of $\mathrm{Co} / \mathrm{Pd}$ multilayers and play a role in getting the desired anisotropy. Comparing the lattice constant of fcc $\mathrm{Pd}$, $3.889 \AA$, and fcc $A u, 4.079 \AA$, we expected an increase of the strain with increased Au concentration. In this paper we examine the magneto-optical(MO) and magnetic properties of $\mathrm{Co} /(\mathrm{Pd}-\mathrm{Au})$ multilayers.

\section{EXPERIMENTAL PROCEDURES}

The multilayers were prepared on a glass substrate by if magnetron sputtering using Ar gas at the gas pressure $5 \mathrm{mTorr}$. All the samples were grown on the Pd buffer layer $300 \AA$ thick at room temperature. The Pd layer thickness was kept constant at $25 \AA$ and that of Co layer was varied in the range 4 to $50 \AA$. The addition of $\mathrm{Au}$ to the $\mathrm{Pd}$ layer was carried out by putting Au chips on the Pd target. The content of Au was determined by energy-dispersive spectrometer(EDS).

The periodic compositional modulations and crystallographic structures of these multilayer were analyzed by small and high angle $\mathrm{x}$-ray diffractions(XRD) using $\mathrm{CuK} \alpha$ radiation. The magnetic properties of the multilayers were investigated by means of a vibrating sample magnetometer (VSM). All measurements were carried out at room temperature. The strength of the magnetic field applied perpendicular and in-plane to the thin film ranged from $-15 \mathrm{kOe}$ 
to $15 \mathrm{kOe}$. The values for the magnetic anisotropy were obtained from the area between the in-plane and perpendicular magnetization curves as measured in VSM. Magneto-optical properties of the sample side were measured in a wavelength range $350-800 \mathrm{~nm}$ using a polar Kerr rotation measurement system at a maximum magnetic field of $15 \mathrm{kOe}$.

\section{RESULTS AND DISCUSSION}

\section{Structure and magnetic property}

Typical high-angle $\mathrm{X}$-ray diffraction patterns for $\mathrm{Co} / \mathrm{Pd}$ $\mathrm{Au}$ ) multilayers are shown in figure 1. In all compositions, the main peaks and satellite peaks can be observed and the existance of periodic structures was confirmed.

Figure 2 illustrates the hysteresis curves for a representative series $\left(t_{C o} \approx 16 \AA\right)$ of $\mathrm{Co} /(\mathrm{Pd}-\mathrm{Au})$ multilayers.

It is clearly seen that with increasing the content of $\mathrm{Au}$ $\left(\mathrm{C}_{\mathrm{Au}}\right)$, multilayered films becomes easier to magnetize in perpendicular fields and increasingly more difficult in parallel fields. But the multilayers become harder to magnetize perpendicular to the film plane, when $\mathrm{C}_{\mathrm{Au}}$ is over 50 at. \%. These results can be explained by a strain contribution to the anisotropy.

As is well known, the magnetic anisotropy energy $K_{\text {eff }} \mathrm{vs}$ the Co layer thickness $t_{c \theta}$ can be phenomenologically described as

$$
K_{e f f} \cdot t=K_{v} \cdot t+2 K_{s}
$$

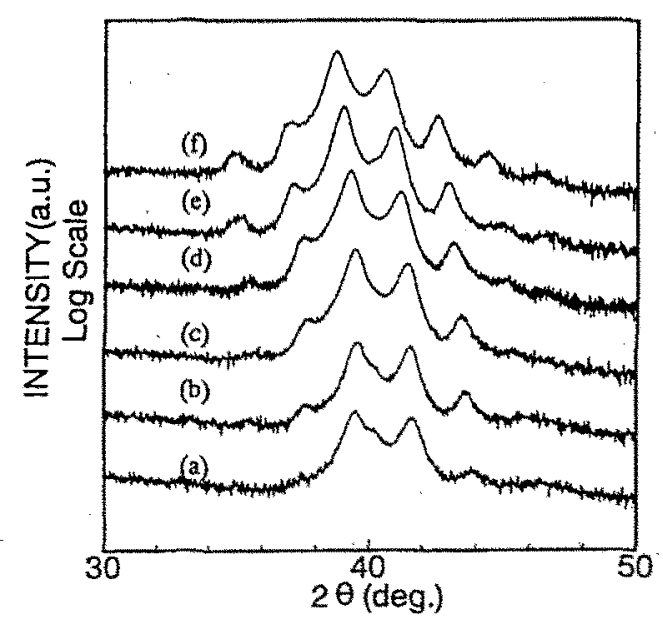

Fig. 1. X-ray diffraction patterns in the high angle region of Co/Pd ${ }_{1-x} A_{x}$ multilayers $\left(t_{C o} \approx 16 \AA\right)$.
(a) $\mathrm{Co} / \mathrm{Pd}$
(b) $\mathrm{Co} / \mathrm{Pd}_{0.955} \mathrm{Au}_{0.045}$
(c) $\mathrm{Co} / \mathrm{Pd}_{0.84} \mathrm{Au}_{0.16}$
(d) $\mathrm{Co} / \mathrm{Pd}_{0.70} \mathrm{Au}_{0.30}$ (e) $\mathrm{Co} / \mathrm{Pd}_{0.56} \mathrm{Au}_{0.44}$
(f) $\mathrm{Co} / \mathrm{Pd}_{0.46} \mathrm{Au}_{0.54}$

where $K_{s}$ refers to the interface anisotropy per unit area and $K_{V}$ is the bulk contributions per unit volume of Co layer.

Figure 3 shows the effecive magnetic anisotropy energy per unit volume of magnetic layer for four compositions with magnetic layer thickness. From the intercept and the slope of linear fits to the data, we can get the interface and volume anisotropies for $\mathrm{Co} / \mathrm{Pd}$ and $\mathrm{Co} / \mathrm{Pd}-\mathrm{Au})$, i.e. $\mathrm{Co} / \mathrm{Pd}_{1-\mathrm{x}} \mathrm{Au}_{\mathrm{x}}(\mathrm{x}=$ $0.045,0.16,0.30)$ muttilayers as listed in Table $\mathrm{I} . \mathrm{Co} /(\mathrm{Pd}-\mathrm{Au})$ multilayers exhibit a perpendicular easy axis of magnetization up to a larger magnetic layer thickness than the Co/Pd multilayer. In figure 3 , by a linear fit, we find that the
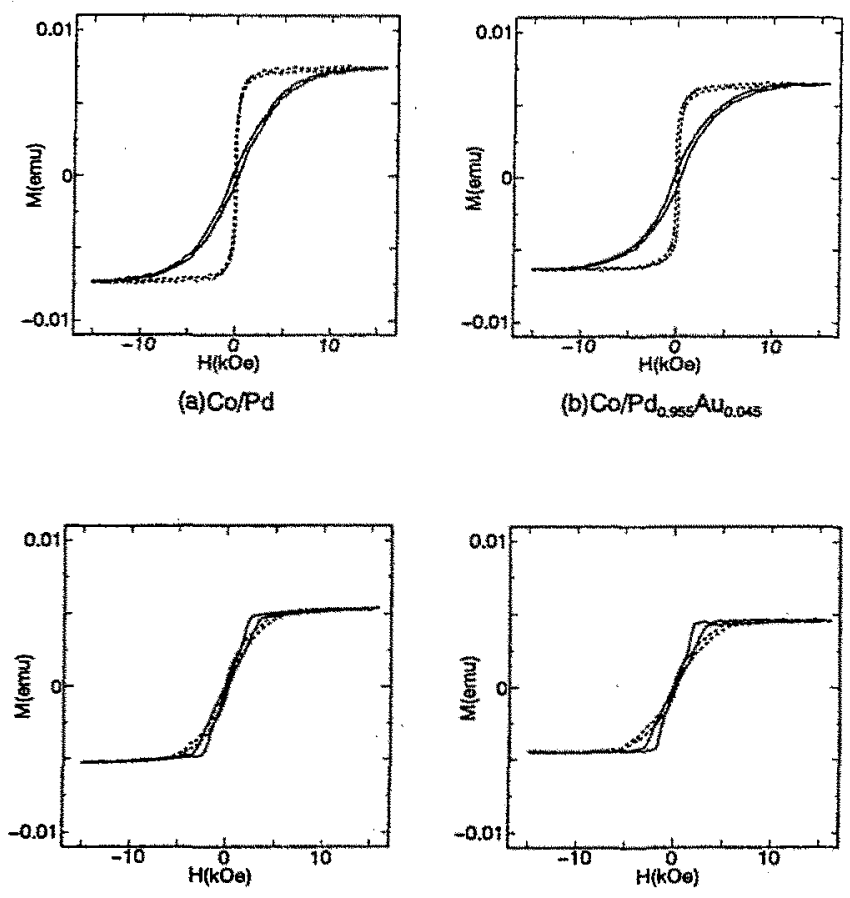

(c) $\mathrm{CO} / \mathrm{Pd}_{0.0 .4} \mathrm{Au}_{0.18}$

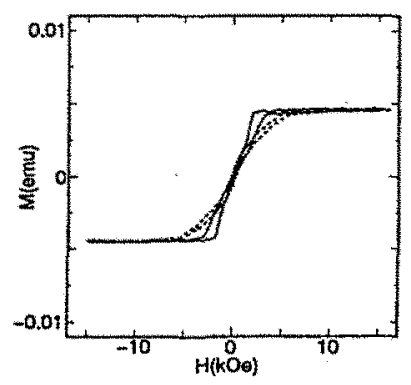

(d) $\operatorname{Co} / P^{P d_{0.70}} A^{A u_{0.30}}$

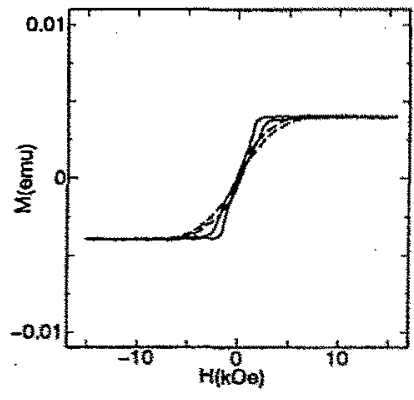

(e) $\mathrm{Co} / \mathrm{Pd}_{\alpha, s} \mathrm{Au}_{0,4.4}$

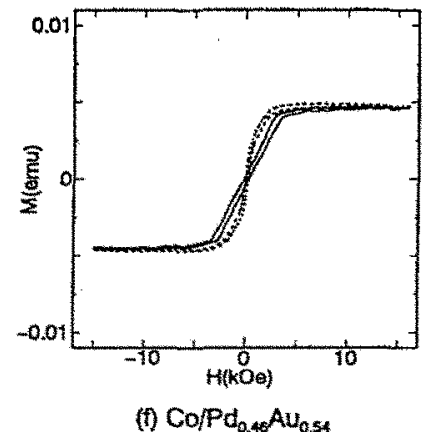

Fig. 2. Magnetization hysteresis loops of a series of $\mathrm{Co} / \mathrm{Pd}-$ Au) multilayers $\left(t_{C o} \approx 16 \AA\right)$. They were measured in fields parallel(--) and perpendicular(-) to the film plane.
(a) $\mathrm{Co} / \mathrm{Pd}$
(b) Co/Pd $0.955 \mathrm{Au}_{0.045}$
(c) $\mathrm{Co} / \mathrm{Pd}_{0.84} A u_{0.16}$
(d) $\mathrm{Co} / \mathrm{Pd}_{0.70} \mathrm{Au}_{0.30}$
(e) $\mathrm{Co} / \mathrm{Pd}_{0.56} \mathrm{Au}_{0.44}$
(f) $\mathrm{Co} / \mathrm{Pd}_{0.46} \mathrm{Au}_{0.54}$ 


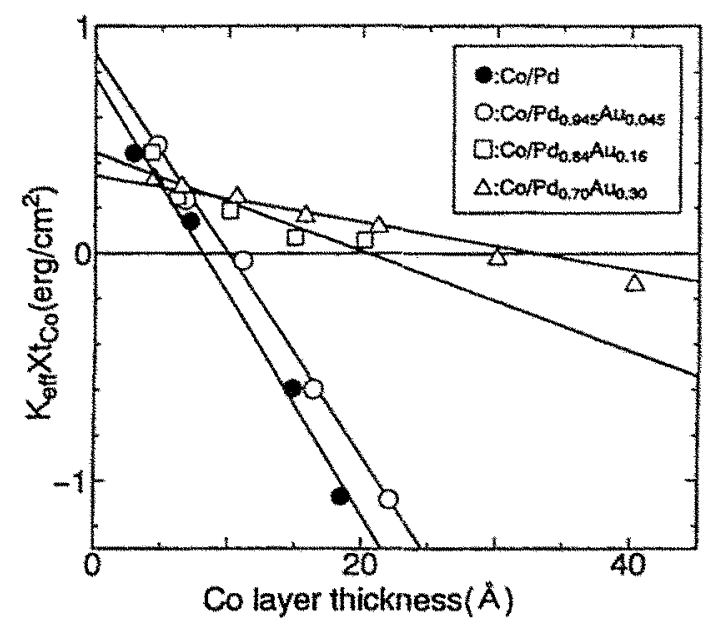

Fig. 3. Plots of effective anisotropy energy $K_{\text {eff }}$ times the Co layer thickness $t_{C o}$ as a function of $t_{C o}$ for $\mathrm{Co} /(\mathrm{Pd}-\mathrm{Au})$ multilayers.

\begin{tabular}{ccc}
\hline Sample & $\begin{array}{c}K_{s} \\
\left(\mathrm{erg}_{\mathrm{cm}}\right)\end{array}$ & $\begin{array}{c}K_{v} \\
\left(1 \mathrm{f}^{6} \mathrm{erg} / \mathrm{cm}^{3}\right)\end{array}$ \\
\hline $\mathrm{Co} / \mathrm{Pd}$ & 0.39 & -9.63 \\
$\mathrm{Co} / \mathrm{Pd}_{0.955} \mathrm{Au}_{0.045}$ & 0.44 & -8.88 \\
$\mathrm{Co}_{\mathrm{Pd}} \mathrm{Pd}_{0.8 \mathrm{Au}_{0.14}}$ & 0.22 & -2.19 \\
$\mathrm{Co} / \mathrm{Pd}_{0.70} \mathrm{Au}_{0.30}$ & 0.17 & -1.05 \\
\hline
\end{tabular}

Tab.1. Interfacial anisotropy energy $K_{S}$ and volume anisotropy energy $K_{y}$ of $\mathrm{Co} /(\mathrm{Pd}-\mathrm{Au})$ multilayers.

transition $\left(K_{\text {eff }}=0\right)$ to a perpendicular easy axis occures at $t_{c o} \approx$ $33 \AA$ for $\mathrm{Co} /\left(\mathrm{Pd}_{0.70} \mathrm{Au}_{0.30}\right)$ multilayer. This is a surprising result. The high quality (111) epitaxial multilayer manufactured by MBE exhibits a perpendicular easy axis of magnetization when the thickness of Co layer is below $24 \AA[9]$. This is believed to be due to the strain effect.

Volume anisotropy energy $K_{V}$ is generally written as

$$
K_{\mathrm{v}}=K_{d}+K_{M C}+K_{M E}
$$

where the first term $K_{d}$ is the demagnetization energy which is the shape contribution, $K_{d}=-2 \pi M_{s}^{2}$ where $M_{s}$ is the saturation magnetization, and $K_{M C}$ is the magnetocrystalline energy of the bulk Co, and the last magnetoelastic energy $\left(K_{M E}\right)$ is the contribution due to stress $(\sigma)$ and the related magnetostriction

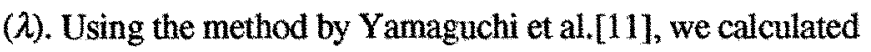
the magnetoelastic energy of $\mathrm{Co} /(\mathrm{Pd}-\mathrm{Au})$ multilayers. If we assume that the magnetic/nonmagnetic interfaces are coherent, $\sigma$ and $K_{M E}$ can be expressed simply by as

\begin{tabular}{cccc}
\hline Sample & $\begin{array}{c}\sigma \\
\left(10^{50} d y n e / \mathrm{cm}^{2}\right)\end{array}$ & $\begin{array}{c}K_{\mathrm{ME}} \\
\left(10^{6} \mathrm{erg} / \mathrm{cm}^{3}\right)\end{array}$ & $\begin{array}{c}K_{y} \\
\left(10^{\circ} \mathrm{erg} / \mathrm{cm}^{3}\right)\end{array}$ \\
\hline $\mathrm{Co} / \mathrm{Pd}$ & 3.26 & 5.38 & -1.83 \\
$\mathrm{Co} / \mathrm{Pd}_{0.055} \mathrm{Au}_{0.045}$ & 3.33 & 5.50 & -1.72 \\
$\mathrm{Co} / \mathrm{Pd}_{0.86} \mathrm{Au}_{0.14}$ & 3.51 & 5.79 & -1.42 \\
$\mathrm{Co} / \mathrm{Pd}_{0.70} \mathrm{Au}_{0.30}$ & 3.72 & 6.14 & -1.08 \\
\hline
\end{tabular}

Tab. II. Strain-induced magnetic anisotropies $K_{M E}$ of $\mathrm{Co} /$ (Pd-Au) multilayers calculated by equation(4).

$$
\begin{gathered}
\sigma=\frac{t_{B} Y_{A} Y_{B}}{t_{A} Y_{A}+t_{B} Y_{B}} \eta \\
K_{M E}=-\frac{3}{2} \lambda_{y j} \sigma
\end{gathered}
$$

where $t_{i}$ and $Y_{i}$ and are thickness of layer $\dot{1}$, the biaxial elastic modulus of (111) planes, respectively and $\eta=\left(a_{B}-a_{A}\right) / a_{A}$ is the lattice mismatch, $\lambda_{111}=-1.1 \times 10^{-4}$ is the magnetostriction coefficient of (111) plane which is the calculated value by the first-principle method[12]. Table II summarizes the straininduced anisotropy energies of $\mathrm{Co} /(\mathrm{Pd}-\mathrm{Au})$ multilayers. This result shows that the increase of $K_{v}$ can be expected by the increase of the strain induced latice mismatch.

It is common practice to write equation (1) as $K_{\text {eff }}=K_{v}+K_{s} t$, assume $K_{s}$ to be constant and consider $K_{\mathrm{V}}$ to be inversely proportional to $t$. However, it has been reported that the magnetoelastic energy due to the in-plane strain play a role to create and stabilize the easy axis perpendicular to the film plane[8][11]. Therefore, magnetoelastic anisotropy can become important.

Furthermore, magnetoelastic energy can contribute to both $K_{s}$ and $K_{v}$ terms in equation (1) because of the stress gradient or periodic stress variation coupled with compositional variation along the film normal. At the interfaces the lattice strain exceeds the measured average value and this will contribute to the interface anisotropy $K_{s}$. The increase of $K_{V}$ with addition of Au can be explained to be due to strain contribution to $K_{v}$. Although other factors such as the atomic mixing or compound formation[13], or the polarization of the Pd layers at the interface[5] may contribute to the perpendicular anisotropy, magnetoelastic effects due to the inplane strain are quite important in understanding the perpendicular anisotropy of magnetic multilayers. 


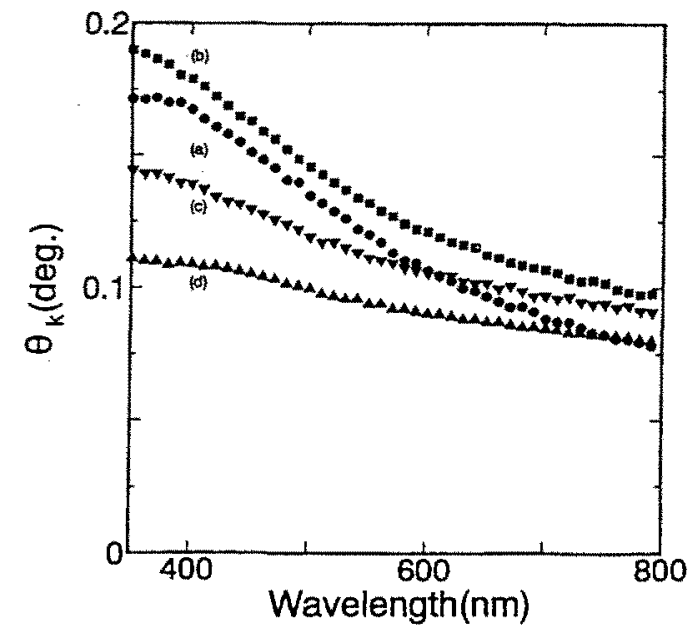

Fig. 4. The polar Kerr rotation spectra of $\mathrm{Co} /(\mathrm{Pd}-\mathrm{Au})$ multilayers $\left(t_{C o} \approx 16 \AA\right)$.
(a) $\mathrm{Co} / \mathrm{Pd}$
$(000)$
(c) $\mathrm{Co} / \mathrm{Pd}_{0.70} \mathrm{Au}_{0.30}(\nabla \nabla \nabla)$
(b) $\mathrm{Co} / \mathrm{Pd}_{0.055} \mathrm{Au}_{0.045}(\mathbf{a} \mathbf{\square})$
(d) $\mathrm{Co} / \mathrm{Pd}_{0.56} \mathrm{Au}_{0.46}(\boldsymbol{\Delta} \mathbf{\Delta} \mathbf{\Delta})$

\section{Magneto-optical(MO) Kerr effect}

Magneto-optical hysteresis loops at $\lambda=400 \mathrm{~nm}$ for these multilayers are measured. As the content of $\mathrm{Au}\left(\mathrm{C}_{\mathrm{Au}}\right)$ increases, the hysteresis loops become easier to magnetize perpendicular to the film plane until $\mathrm{C}_{\mathrm{Au}}=44 \mathrm{at} \%$. But the multilayers become harder to magnetize perpendicular to the film plane, when $C_{A u}$ is over 50 at. \%. These results is consistent with the results in Figure 2.

The spectra of the polar Kerr rotation angle $\theta_{k}$, measured in a magnetic field of $15 \mathrm{kOe}$, i.e., in the saturation state, are shown in Figure 4. The Kerr effect increases at $\mathrm{C}_{\mathrm{Au}}=4.5 \mathrm{at} . \%$ compared to $\mathrm{Co} / \mathrm{Pd}$ multilayers, but decreases with increasing $\mathrm{Au}$ content. The shape of the spectra is similar to Co/Pd multilayers. But the enhancement in the polar Kerr rotation near the shorter-wavelength range(about $400 \mathrm{~nm}$ ) in $\mathrm{Co} / \mathrm{Pd}$ multilayers decreases with increasing Au content. The decrease of $\theta_{k}$ with increasing Au content can be explained to be due to reduction of polarized $\mathrm{Pd}$.

\section{CONCLUSIONS}

We have used magnetron sputtering to deposit $\mathrm{Co} /(\mathrm{Pd}-$ $\mathrm{Au})$ multilayers onto a glass substrates. $\mathrm{Co} /(\mathrm{Pd}-\mathrm{Au})$ multilayers exhibit a magnetic easy axis perpendicular to the film plane up to a larger magnetic layer thickness than $\mathrm{Co} / \mathrm{Pd}$ multilayer. Especially, $\mathrm{Co} /\left(\mathrm{Pd}_{0.70} \mathrm{Au}_{0.30}\right)$ multilayers became perpendicular at $33 \AA$. The relation between magnetic anisotropy and lattice strain can be explained as magnetoelastic energy due to lattice mismatch between the adjacent layers. The role of magnetoelastic energy due to the in-plane strain in the system is pointed out and discussed. We also investigated the magneto-optical(MO) polar Kert effect of these multilayers. The polar Kerr rotation angle $\theta_{\mathrm{k}}$ increases at $\mathrm{C}_{\mathrm{Au}}=4.5 \mathrm{at} . \%$ compared to $\mathrm{Co} / \mathrm{Pd}$ multilayers, but decreases with increasing $\mathrm{Au}$ content.

\section{ACKNOWLEDGMENTS}

We would like to thank Y. Sabi and M. Kaneko of Sony Corporation for help in magneto-optical measurements and Oki Electrics Corporation for help in the experiment. This work was supported by a Grant-in-Aid for Scientific Research from the Ministry of Education, Science and Culture, No.06452325.

\section{REFERENCES}

[1] T. Katayama, H. Awano, Y. Nishihara, and N. Koshizuka : IEEE Trans. Magn., 23, 2949 (1987).

[2] S. Hashimoto and Y. Ochiai : J. Magn. Magn. Mater., 88, 211 (1990).

[3] P. F. Carcia, A. D. Meinhaldt, and A. Suna : Appl. Phys. Lett., 47, 178(1985).

[4] P. F. Carcia: J. Appl. Phys., 63, 5066(1988).

[5] F. J. A. den Broeder, H. C. Donkersloot, H. J. G. Draasima , and W. J. M. de Jonge : J. Appl. Phys., 61, 4317(1987).

[6] F. J. A. den Broeder, D. Kupier, A. P. van de Mosselaer, and W. Hoving : Phys. Rev. Lett., 60, 2769(1988).

[7] L. Néel : J. Phys. Radium, 15, 225(1954).

[8] B. Zhang, Kannan M. Krishnan, C. H. Lee, and R. F. C. Farrow : J. Appl. Phys., 73, 6198(1993).

[9] Brad N. Engel, Craig D. England, Robert A. Van Leeuwen, Michael H. Wiedmann, and Charles M. Falco : J. Appl. Phys., 70, 5873(1991).

[10] Brad N. Engel, Craig D. England, Robert A. Van Leeuwen, Michael H. Wiedmann, and Charles M. Falco : Phys. Rev. Lett., 67, 1910(1991).

[11] A. Yamaguchi, S. Ogu, W.-H. Soe, and R. Yamamoto: Appl. Phys. Lett., 62, 1020 (1993).

[12] K. Kyuno : "Theoretical stydy on the magnetic anisotropy of metallic multilayer", Ph. D. Thesis, Univ. Tokyo, Japan(1994).

[13] N.-H. Cho, Kannan M. Krishnan, C. A. Lucas, and R. F. C. Farrow : J. Appl. Phys., 72, 5799(1992). 